VoL. 67 (2003) [103-114]

\title{
ON SUMS OF CERTAIN TRIGONOMETRIC SERIES
}

\author{
Minking EIE AND YaO LIN ONG
}

Let $N$ be a fixed positive integer. In this paper, we apply a newly developed method of Eie and Lai to the evaluation of certain infinite trigonometric series. Indeed, we are able to express the sums of these series in terms of Bernoulli polynomials. That is, we obtain several new Bernoulli identities. A similar method leads to Ramanujan's identities.

\section{SPECIAL VALUES OF $L$-FUnCTIONS AT POSITIVE INTEgers}

Let $\chi$ be a Dirichlet character modulo $N$ and let

$$
L(s, \chi)=\sum_{n=1}^{\infty} \frac{\chi(n)}{n^{s}}, \quad \operatorname{Re} s>1
$$

be the corresponding Dirichlet $L$-function. Through the functional equation for $L(s, \chi)$ and its special values at negative integers, it is possible to evaluate $L(s, \chi)$ at positive integers. The results are as follows.

(1) If $\chi(-1)=1$, then for any positive integer $m$,

$$
L(2 m, \chi)=\frac{(-1)^{m}}{(2 m) !}\left(\frac{2 \pi}{N}\right)^{2 m} \tau(\chi)\left(-B_{\bar{\chi}}^{2 m}\right) .
$$

(2) If $\chi(-1)=-1$, then for any positive integer $m$,

$$
L(2 m+1, \chi)=(-i) \frac{(-1)^{m}}{(2 m+1) !}\left(\frac{2 \pi}{N}\right)^{2 m+1} \tau(\chi)\left(-B_{\bar{\chi}}^{2 m+1}\right),
$$

where

$$
\tau(\chi)=\sum_{a=1}^{N} \chi(a) e^{2 \pi i a / N}
$$

is the Gaussian sum and $B_{\chi}^{n}(n=0,1,2, \ldots)$ are the generalised Bernoulli numbers defined by

$$
\sum_{j=1}^{N} \frac{\chi(j) t e^{j t}}{e^{N t}-1}=\sum_{n=0}^{\infty} B_{\chi}^{n} \frac{t^{n}}{n !}|t|<\frac{2 \pi}{N} .
$$

Received 24th June, 2002

This work was supported by the Department of Mathematics, National Chung Cheng University and by the Natiaonal Science Fundation of Taiwan, Republic of China.

Copyright Clearance Centre, Inc. Serial-fee code: 0004-9727/03 \$A2.00+0.00. 
In this paper, the role of the Dirichlet character $\chi(n)$ will be replaced by trigonometric functions such as $\tan (n \pi / N), \cot (n \pi / N), \sec (n \pi / N), \csc (n \pi / N)$ whenever they are defined. We obtain the following new identities.

(1)

$$
\sum_{\substack{n=1 \\ n \neq 00}}^{\infty} \frac{\cot (n \pi / N)}{n^{2 m+1}}=\frac{(-1)^{m+1}(2 \pi)^{2 m+1}}{N(2 m+1) !} \sum_{j=1}^{N-2}(N-j-1) B_{2 m+1}\left(\frac{j}{N}\right) .
$$

(2) If $N$ is odd, then

$$
\sum_{n=1}^{\infty} \frac{\tan (n \pi / N)}{n^{2 m+1}}=\frac{(-1)^{m}(2 \pi)^{2 m+1}}{2(2 m+1) !} \sum_{j=1}^{N}(-1)^{j} B_{2 m+1}\left(\frac{j}{N}\right)
$$

(3) If $N$ is even, then

$$
\sum_{\substack{n=1 \\ n \neq N / 2(\bmod N)}}^{\infty} \frac{\tan (n \pi / N)}{n^{2 m+1}}=\frac{(-1)^{m}(2 \pi)^{2 m+1}}{N(2 m+1) !} \sum_{j=1}^{N}(-1)^{j}(N-j-1) B_{2 m+1}\left(\frac{j}{N}\right) .
$$

(4)

$$
\begin{aligned}
& \sum_{\substack{n=1 \\
n \neq 0}}^{\infty} \frac{\csc (n \pi / N)}{n^{2 m+1}} \\
& \quad=\frac{(-1)^{m+1}(2 \pi)^{2 m+1}}{N(2 m+1) !} \sum_{j=0}^{N-2}(N-j-1) B_{2 m+1}\left(1+\frac{1}{2 N}+\frac{j}{N}\right) \\
& \quad+\frac{(-1)^{m+1}(2 \pi)^{2 m+1}\left(1-2^{-(2 m+1)}\right)}{N^{2 m+1}(2 m) !} \frac{B_{2 m+2}}{2 m+2} \\
& \quad+\frac{(-1)^{m+1}(2 \pi)^{2 m+1}}{N^{2 m+1}(2 m) !}\left[\frac{1}{2 m+2} B_{2 m+2}\left(N+\frac{1}{2}\right)-\frac{N-(1 / 2)}{2 m+1} B_{2 m+1}\left(N+\frac{1}{2}\right)\right]
\end{aligned}
$$

(5) If $N$ is odd, then

$$
\begin{aligned}
\sum_{\substack{n=1 \\
n \neq 0}}^{\infty} \frac{\sec (n \pi / N)}{n^{2 m+2}} & =\frac{(-1)^{m}(2 \pi)^{2 m+2}}{2(2 m+1) !} \sum_{j=0}^{N-1}(-1)^{j} B_{2 m+2}\left(1+\frac{1}{2 N}+\frac{j}{N}\right) \\
& +\frac{(-1)^{m}(4 \pi)^{2 m+2}}{4 N^{2 m+1}(2 m+2) !}\left[B_{2 m+2}\left(\frac{N}{2}+\frac{1}{4}\right)-B_{2 m+2}\left(\frac{N}{2}+\frac{3}{4}\right)\right]
\end{aligned}
$$

(6) If $N$ is even, then 


$$
\begin{aligned}
& \sum_{\substack{n=1 \\
n \neq N / 2(\bmod N)}}^{\infty} \frac{\sec (n \pi / N)}{n^{2 m+2}} \\
& =\frac{(-1)^{m}(2 \pi)^{2 m+2}}{N(2 m+2) !} \sum_{j=0}^{N-2}(-1)^{j} B_{2 m+2}\left(1+\frac{1}{2 N}+\frac{j}{N}\right) \\
& \quad+\frac{(-1)^{m+1}(2 \pi)^{2 m+2}}{N^{2 m+2}(2 m+1) !}\left[\left(\frac{B_{2 m+3}(N+(1 / 2))}{2 m+3}-2^{2 m+3} \frac{B_{2 m+3}(N / 2+1 / 4)}{2 m+3}\right)\right. \\
& \left.\quad+\left(\frac{1}{2}-N\right)\left(\frac{B_{2 m+2}(N+(1 / 2))}{2 m+2}-2^{2 m+2} \frac{B_{2 m+2}((N / 2)+(1 / 4))}{2 m+2}\right)\right] .
\end{aligned}
$$

It is not difficult to derive all these identities once we apply the newly developed method in [2] to the zeta functions associated with rational functions of the form

$$
F(T)=\frac{P(T)}{\left(1-T^{m_{1}}\right)\left(1-T^{m_{2}}\right) \cdots\left(1-T^{m_{r}}\right)}
$$

where $m_{1}, m_{2}, \cdots, m_{r}$ are complex numbers with positive real parts and $P(T)$ is a finite linear combination of $T^{\beta}, \operatorname{Re} \beta>0$, with complex numbers. By specifying $F(T)$ to be

$$
\frac{1}{(1-T)\left(1-T^{N}\right)} \quad \text { and } \quad \frac{1}{(1+T)\left(1-T^{N}\right)}
$$

or

$$
\frac{T^{(1 / 2)+N}}{(1-T)\left(1-T^{N}\right)} \text { and } \frac{T^{(1 / 2)+N}}{(1+T)\left(1-T^{N}\right)}
$$

we obtain our identities. Similar arguments can be used to derive identities in [1, Chapter 14 of Part II]. As a consequence, we have the following identities for any odd integer $n$ :

$$
\begin{aligned}
& \text { (1) } \sum_{k=1}^{\infty}(\operatorname{coth}(\pi k)) /\left(k^{2 n+1}\right)=2^{2 n} \pi^{2 n+1} \sum_{k=0}^{n+1}(-1)^{k+1}\left(B_{2 k} B_{2 n+2-2 k}\right) /((2 k) \\
& !(2 n+2-2 k) !) \\
& \text { (2) } \sum_{k=1}^{\infty}\left((-1)^{k+1} \operatorname{csch}(\pi k)\right) /\left(k^{2 n+1}\right)=2^{2 n} \pi^{2 n+1} \sum_{k=0}^{n+1}(-1)^{k}\left(B_{2 k}(1 / 2) B_{2 n+2-2 k}\right. \\
& (1 / 2)) /((2 k) !(2 n+2-2 k) !),
\end{aligned}
$$

\section{ZETA-FUNCTIONS ASSOCIATED WITH RATIONAL FUNCTIONS}

Consider the rational function of the form

$$
F(T)=\frac{P(T)}{\left(1-T^{m_{1}}\right)\left(1-T^{m_{2}}\right) \cdots\left(1-T^{m_{r}}\right)}
$$


where $m_{1}, m_{2}, \cdots, m_{r}$ are positive integers and $P(T)$ is a polynomial in $T$ (with complex coefficients) of degree less than $m_{1}+m_{2}+\cdots+m_{r}$. For $|T|<1, F(T)$ has a power series expansion

$$
F(T)=\sum_{k=0}^{\infty} a(k) T^{k}
$$

The value of $a(k)$ is determined via the residue theorem as

$$
a(k)=\frac{1}{2 \pi i} \int_{\mathcal{C}} \frac{F(z) d z}{z^{k+1}}
$$

where $\mathcal{C}$ is a sufficiently small circle centred at the origin going counterclockwise.

The zeta function $Z_{F}(s)$ associated with $F(T)$ is then given by

$$
Z_{F}(s)=\sum_{k=1}^{\infty} a(k) k^{-s}, \quad \operatorname{Re} s>r
$$

which is determined by $F(T)$ uniquely through a Mellin transform

$$
Z_{F}(s) \Gamma(s)=\int_{0}^{\infty} t^{s-1}\left[F\left(e^{-t}\right)-F(0)\right] d t
$$

for $\operatorname{Re} s>r$, where $\Gamma(s)$ is the classical gamma function defined by

$$
\Gamma(s)=\int_{0}^{\infty} t^{s-1} e^{-t} d t, \quad \operatorname{Re} s>0
$$

The possible poles of $F\left(e^{-z}\right)$ lie in the imaginary axis of the complex plane. By a direct verification, we can find a sequence of contours $C_{N}(N=1,2, \ldots)$ such that the following conditions hold:

(1) $C_{N}$ is the rectangle with vertices $x_{N}+i y_{N}, x_{N}-i y_{N},-x_{N}+i y_{N}$, $-x_{N}-i y_{N}, x_{N}>0, y_{N}>0$, and its direction going counterclockwise.

(2) $\lim _{N \rightarrow \infty} x_{N}=\lim _{N \rightarrow \infty} y_{N}=+\infty$.

(3) $C_{N}$ does not pass through any pole of $F\left(e^{-z}\right)$.

(4) $\max _{z \in C_{N}}\left|F\left(e^{-z}\right)\right|$ is bounded by a constant independent of $N$.

It follows that for any positive integer $n$,

$$
\lim _{N \rightarrow \infty} \frac{1}{2 \pi i} \int_{C_{N}} z^{-(n+1)} F\left(e^{-z}\right) d z=0
$$


This also implies that the residue of $z^{-(n+1)} F\left(e^{-z}\right)$ at $z=0$ is equal to the negative of the sum of residues of $z^{-(n+1)} F\left(e^{-z}\right)$ at $z=2 k \pi i / m_{j}, k \in \mathbb{Z}, k \neq 0, j=1, \ldots, r$. That is,

$$
\begin{aligned}
\frac{1}{2 \pi i} \int_{|z|=\varepsilon} z^{-(n+1)} F\left(e^{-z}\right) & d z \\
= & -\sum_{k \neq 0}\left\{\text { Residue of } z^{-(n+1)} F\left(e^{-z}\right) \text { at } z=2 k \pi i / m_{j}\right\}
\end{aligned}
$$

Note that residue theory shows the left hand side of (2.1) is a constant multiple of $Z_{F}(-n)$, and gives the right hand side of (2.1) as an infinite series. This produces an identity among the Bernoulli numbers and the sums of infinite series. On the other hand, we are able to decompose $F(T)$ into the sum of its partial fractions, so that $Z_{F}(S)$ can be expressed as a sum of Riemann zeta functions and Hurwitz zeta functions. For example, if

$$
F(T)=\frac{1}{\left(1-T^{2}\right)\left(1-T^{3}\right)}
$$

one can decompose $F$ into

$$
\begin{aligned}
\frac{1}{6(1-T)^{2}}+ & \frac{1}{4(1-T)}+\frac{1-T}{4\left(1-T^{2}\right)}+\frac{1-T}{3\left(1-T^{3}\right)} \\
& =\frac{1}{6} \sum_{k=0}^{\infty}(k+1) T^{k}+\frac{1}{4} \sum_{k=0}^{\infty} T^{k}+\frac{1}{4} \sum_{k=0}^{\infty}(1-T) T^{2 k}+\frac{1}{3} \sum_{k=0}^{\infty}(1-T) T^{3 k}
\end{aligned}
$$

It follows that

$$
Z_{F}(s)=\frac{1}{6}[\zeta(s-1)+\zeta(s)]+\frac{1}{4} \zeta(s)+\frac{2^{-s}}{4}\left[\zeta(s)-\zeta\left(s ; \frac{1}{2}\right)\right]+\frac{3^{-s}}{3}\left[\zeta(s)-\zeta\left(s ; \frac{1}{3}\right)\right] .
$$

It is well known that Riemann zeta functions and Hurwitz zeta functions both have analytic continuations in the whole complex plane and

$$
\zeta(1-m)=(-1)^{m-1} \frac{B_{m}}{m}, \quad \zeta(1-m ; \delta)=-\frac{B_{m}(\delta)}{m} \quad(m=1,2,3, \ldots) .
$$

Therefore, $Z_{F}(s)$ has its analytic continuation and its special values at negative integers can be given explicitly in terms of Bernoulli numbers and Bernoulli polynomials.

Now we are ready to derive our first identity.

THEOREM I. For positive integers $m$ and $N$ with $N \geqslant 3$,

$$
\sum_{\substack{n=1 \\ n \neq 0}}^{\infty} \frac{\cot (n \pi / N)}{n^{2 m+1}}=\frac{(-1)^{m+1}(2 \pi)^{2 m+1}}{N(2 m+1) !} \sum_{j=1}^{N-2}(N-j-1) B_{2 m+1}\left(\frac{j}{N}\right) .
$$


PROOF: Consider the rational function

$$
F(T)=\frac{1}{(1-T)\left(1-T^{N}\right)}
$$

From the partial fractions decomposition

$$
F(T)=\frac{1}{N(1-T)^{2}}+\frac{(N-1)+(N-2) T+\cdots+T^{N-2}}{N\left(1-T^{N}\right)}
$$

we conclude that

$$
Z_{F}(s)=\frac{1}{N}[\zeta(s-1)+\zeta(s)]+\frac{N-1}{N} N^{-s} \zeta(s)+\sum_{j=1}^{N-2}(N-j-1) N^{-s-1} \zeta\left(s, \frac{j}{N}\right)
$$

Consequently for any positive integer $m$, we get

$$
Z_{F}(-2 m)=-\frac{1}{N} \frac{B_{2 m+2}}{2 m+2}-\frac{N^{2 m-1}}{2 m+1} \sum_{j=1}^{N-2}(N-j-1) B_{2 m+1}\left(\frac{j}{N}\right)
$$

On the other hand, we have for $\operatorname{Re} s>2$ that

$$
\begin{aligned}
Z_{F}(s) \Gamma(s) & =\int_{0}^{\infty} t^{s-1}\left[\frac{1}{\left(1-e^{-t}\right)\left(1-e^{-N t}\right)}-1\right] d t \\
& =\int_{0}^{\infty} t^{s-1}\left[\frac{1}{\left(e^{t}-1\right)\left(e^{N t}-1\right)}+\frac{1}{e^{t}-1}+\frac{1}{e^{N t}-1}\right] d t \\
& =\int_{0}^{\infty} \frac{t^{s-1} d t}{\left(e^{t}-1\right)\left(e^{N t}-1\right)}+\left(N^{-s}+1\right) \zeta(s) \Gamma(s)
\end{aligned}
$$

Let $L(\varepsilon)$ be the key contour in the complex plane, consisting of the intervals $[\varepsilon,+\infty)$ twice in opposite directions and the circle $|z|=\varepsilon$ with the direction going counterclockwise. Then for $\operatorname{Re} s>2$,

$$
\begin{aligned}
\int_{L(\varepsilon)} \frac{z^{s-1} d z}{\left(e^{z}-1\right)\left(e^{N z}-1\right)} & \\
& =\left(e^{2 \pi i s}-1\right) \int_{\varepsilon}^{\infty} \frac{t^{s-1} d t}{\left(e^{t}-1\right)\left(e^{N t}-1\right)}+\int_{|z|=\varepsilon} \frac{z^{s-1} d z}{\left(e^{z}-1\right)\left(e^{N z}-1\right)}
\end{aligned}
$$

As $\varepsilon \rightarrow 0$, the second integral on the right hand side approaches 0 . This gives

$$
\lim _{\varepsilon \rightarrow 0} \int_{L(\varepsilon)} \frac{z^{s-1} d z}{\left(e^{z}-1\right)\left(e^{N z}-1\right)}=\left(e^{2 \pi i s}-1\right) \int_{0}^{\infty} \frac{t^{s-1} d t}{\left(e^{t}-1\right)\left(e^{N t}-1\right)}
$$


Still, the contour integral is independent of $\varepsilon$, so we can eliminate the limit. Thus for Re $s>2$ and $s \notin \mathbb{Z}$, we have

$$
Z_{F}(s) \Gamma(s)=\left(e^{2 \pi i s}-1\right)^{-1} \int_{L(\varepsilon)} \frac{z^{s-1} d z}{\left(e^{z}-1\right)\left(e^{N z}-1\right)}+\left(N^{-s}+1\right) \zeta(s) \Gamma(s) .
$$

With the help of the functional equation

$$
\Gamma(s) \Gamma(1-s)=\frac{\pi}{\sin \pi s}=\frac{2 \pi i e^{\pi i s}}{e^{2 \pi i s}-1}
$$

we obtain

$$
Z_{F}(s)-\left(N^{-s}+1\right) \zeta(s)=\Gamma(1-s) e^{-\pi i s} \frac{1}{2 \pi i} \int_{L(\varepsilon)} \frac{z^{s-1} d z}{\left(e^{z}-1\right)\left(e^{N z}-1\right)}
$$

This gives the analytic continuation of $Z_{F}(s)$. Now for $s=-2 m$, the integrations along $[\varepsilon,+\infty)$ twice in opposite directions cancel each other. Hence we have

$$
\begin{aligned}
Z_{F}(-2 m) & =(2 m) ! \frac{1}{2 \pi i} \int_{|z|=\varepsilon} \frac{z^{-(2 m+1)} d z}{\left(e^{z}-1\right)\left(e^{N z}-1\right)} \\
& =-(2 m) ! \sum_{k \neq 0}\left[\text { Residue of } z^{-(2 m+1)} F\left(e^{z}\right) \text { at } z=2 k \pi i / N\right] \\
& =\frac{(2 m+1) !}{N} \sum_{k \neq 0} \frac{1}{(2 k \pi i)^{2 m+2}}-\frac{(2 m) !}{N} \sum_{\substack{k \neq 0 \\
k \neq 0(\bmod N)}}\left(\frac{N}{2 k \pi i}\right)^{2 m+1} \frac{1}{e^{2 k \pi i / N}-1} \\
& =\frac{2}{N} \frac{(-1)^{m+1}(2 m+1) ! \zeta(2 m+2)}{(2 \pi)^{2 m+2}}+\frac{(-1)^{m}(2 m) !}{N(2 \pi)^{2 m+1}} \sum_{j=1}^{N-1} \cot \frac{j \pi}{N} \zeta\left(2 m+1, \frac{j}{N}\right) .
\end{aligned}
$$

By applying the well-known formula

$$
-\frac{B_{2 m+2}}{2 m+2}=\frac{2(-1)^{m+1}(2 m+1) ! \zeta(2 m+2)}{(2 \pi)^{2 m+2}}
$$

we obtain

$$
\sum_{j=1}^{N-1} \cot \frac{j \pi}{N} \zeta\left(2 m+1, \frac{j}{N}\right)=\frac{(-1)^{m+1}(2 \pi)^{2 m+1} N^{2 m}}{(2 m+1) !} \sum_{j=1}^{N-2}(N-j-1) B_{2 m+1}\left(\frac{j}{N}\right) .
$$

This is equivalent to our required identity. 
If $N$ is odd, choose $F(T)$ to be

$$
F(T)=\frac{1}{(1+T)\left(1-T^{N}\right)}
$$

Then the decomposition

$$
\frac{1}{2(1+T)}+\frac{1-T+T^{2}-\ldots+T^{N-1}}{2\left(1-T^{N}\right)}
$$

gives

$$
Z_{F}(s)=2^{-1-s}\left[\zeta(s)-\zeta\left(s, \frac{1}{2}\right)\right]+\frac{N^{-s}}{2} \zeta(s)+\frac{N^{-s}}{2} \sum_{j=1}^{N-1}(-1)^{j-1} \zeta\left(s, \frac{N-j}{N}\right)
$$

It follows that

$$
Z_{F}(-2 m)=\frac{N^{2 m}}{2(2 m+1)} \sum_{j=1}^{N-1}(-1)^{j} B_{2 m+1}\left(\frac{N-j}{N}\right)
$$

On the other hand, we also have

$$
Z_{F}(-2 m)=\frac{(-1)^{m+1}(2 m) !}{N(2 \pi)^{2 m+1}} \sum_{j=1}^{N} \tan \frac{j \pi}{N} \zeta\left(2 m+1, \frac{j}{N}\right)
$$

Consequently, we have the following identity.

ThEOREM II. For any positive integer $m$ and odd integers $N$ with $N \geqslant 3$,

$$
\sum_{n=1}^{\infty} \frac{\tan (n \pi / N)}{n^{2 m+1}}=\frac{(-1)^{m}(2 \pi)^{2 m+1}}{2(2 m+1) !} \sum_{j=1}^{N}(-1)^{j} B_{2 m+1}\left(\frac{j}{N}\right)
$$

If $N$ is even, another decomposition

$$
F(T)=\frac{1}{(1+T)\left(1-T^{N}\right)}=\frac{1}{N(1+T)^{2}}+\frac{(N-1)-(N-2) T+\ldots+T^{N-2}}{N\left(1-T^{N}\right)} .
$$

gives the following identity immediately.

TheOREM III. For any positive integer $m$ and even integers $N$ with $N \geqslant 3$,

$$
\sum_{n=1, n \neq N / 2(\bmod N)}^{\infty} \frac{\tan (n \pi / N)}{n^{2 m+1}}=\frac{(-1)^{m}(2 \pi)^{2 m+1}}{N(2 m+1) !} \sum_{j=1}^{N}(-1)^{j}(N-j-1) B_{2 m+1}\left(\frac{j}{N}\right)
$$




\section{Further Results}

If we consider the function

$$
F(T)=\frac{T^{(1 / 2)+N}}{(1-T)\left(1-T^{N}\right)}
$$

and use the decomposition

$$
F(T)=\frac{T^{(1 / 2)+N}}{N(1-T)^{2}}+\frac{T^{(1 / 2)+N}\left[(N-1)+(N-2) T+\ldots+T^{N-2}\right]}{N\left(1-T^{N}\right)},
$$

then we get the following identity.

THEOREM IV. For positive integers $m$ and $N$ with $N \geqslant 3$,

$$
\begin{aligned}
& \sum_{n=1 n \neq 0(\bmod N)}^{\infty} \frac{\csc (n \pi / N)}{n^{2 m+1}} \\
& =\frac{(-1)^{m+1}(2 \pi)^{2 m+1}}{N(2 m+1) !} \sum_{j=0}^{N-2}(N-j-1) B_{2 m+1}\left(1+\frac{1}{2 N}+\frac{j}{N}\right) \\
& \quad+\frac{(-1)^{m+1}(2 \pi)^{2 m+1}\left(1-2^{-(2 m+1)}\right)}{N^{2 m+1}(2 m) !} \frac{B_{2 m+2}}{2 m+2} \\
& \quad+\frac{(-1)^{m+1}(2 \pi)^{2 m+1}}{N^{2 m+1}(2 m) !}\left[\frac{1}{2 m+2} B_{2 m+2}\left(N+\frac{1}{2}\right)-\frac{N-(1 / 2)}{2 m+1} B_{2 m+1}\left(N+\frac{1}{2}\right)\right] .
\end{aligned}
$$

In a similar way, the following two identities are easily obtained by considering the function

$$
F(T)=\frac{T^{(1 / 2)+N}}{(1+T)\left(1-T^{N}\right)}
$$

with its decomposition

$$
F(T)=\frac{T^{(1 / 2)+N}}{2(1+T)}+\frac{T^{(1 / 2)+N}\left[1-T+T^{2}-\ldots+T^{N-1}\right]}{2\left(1-T^{N}\right)}
$$

when $N$ is odd, and

$$
F(T)=\frac{T^{(1 / 2)+N}}{N(1+T)^{2}}+\frac{T^{(1 / 2)+N}\left[(N-1)-(N-2) T+\ldots+T^{N-2}\right]}{N\left(1-T^{N}\right)}
$$

when $N$ is even. 
THEOREM V. For any positive integer $m$ and odd integers $N$ with $N \geqslant 3$,

$$
\begin{aligned}
\sum_{\substack{n=1 \\
n \neq 0(\bmod N)}}^{\infty} \frac{\sec (n \pi / N)}{n^{2 m+2}}= & \frac{(-1)^{m}(2 \pi)^{2 m+2}}{2(2 m+2) !} \sum_{j=0}^{N-1}(-1)^{j} B_{2 m+2}\left(1+\frac{1}{2 N}+\frac{j}{N}\right) \\
& +\frac{(-1)^{m}(4 \pi)^{2 m+2}}{4 N^{2 m+1}(2 m+2) !} B_{2 m+2}\left(\frac{N}{2}+\frac{1}{4}\right)-B_{2 m+2}\left(\frac{N}{2}+\frac{3}{4}\right) .
\end{aligned}
$$

TheOREM VI. For any positive integer $m$ and even integers $N$ with $N \geqslant 3$,

$$
\begin{aligned}
& \sum_{\substack{n=1 \\
n \neq N / 2}}^{\infty} \frac{\sec (n \pi / N)}{n^{2 m+2}} \\
= & \frac{(-1)^{m}(2 \pi)^{2 m+2}}{N(2 m+2) !} \sum_{j=0}^{N-2}(-1)^{j} B_{2 m+2}\left(1+\frac{1}{2 N}+\frac{j}{N}\right) \\
& +\frac{(-1)^{m+1}(2 \pi)^{2 m+2}}{N^{2 m+2}(2 m+1) !}\left[\left(\frac{B_{2 m+3}(N+(1 / 2))}{2 m+3}-2^{2 m+3} \frac{B_{2 m+3}((N / 2)+(1 / 4))}{2 m+3}\right)\right. \\
& \left.+\left(\frac{1}{2}-N\right)\left(\frac{B_{2 m+2}(N+(1 / 2))}{2 m+2}-2^{2 m+2} \frac{B_{2 m+2}((N /) 2+(1 / 4))}{2 m+2}\right)\right] .
\end{aligned}
$$

\section{Two Ramanujan's IDENTITIES}

As mentioned in [2], it is possible to generalise the previous argument to include the case

$$
F(T)=\frac{P(T)}{\left(1-T^{m_{1}}\right) \ldots \ldots\left(1-T^{m_{r}}\right)}
$$

where

(1) $m_{1}, \ldots, m_{r}$ are not necessary positive integers, but are complex numbers with Re $m_{j}>0$;

(2) $P(T)$ is not necessary a polynomial, but a finite linear combination of $T^{\beta}$ with complex numbers and $\operatorname{Re} \beta \geqslant 0$.

As an example we derive two identities in [1, Chapter 14 of part II].

EXAMPLE. For any odd integer $n$, we have

$$
\begin{array}{ll}
\text { (1) } & \sum_{k=1}^{\infty}\left(\operatorname{coth}(\pi k) / k^{2 n+1}\right)=2^{2 n} \pi^{2 n+1} \sum_{k=0}^{n+1}(-1)^{k+1}\left(B_{2 k} /(2 k) !\right)\left(B_{2 n+2-2 k} /\right. \\
& (2 n+2-2 k) !) . \\
\text { (2) } & \sum_{k=1}^{\infty}\left((-1)^{k+1} \operatorname{csch}(\pi k) / k^{2 n+1}\right)=2^{2 n} \pi^{2 n+1} \sum_{k=0}^{n+1}(-1)^{k}\left(B_{2 k}(1 / 2) /(2 k) !\right) \\
& \left(B_{2 n+2-2 k}(1 / 2) /(2 n+2-2 k) !\right) .
\end{array}
$$


Proof: For $0<u, v<1$ and $\varepsilon>0$, consider the function

$$
F(T)=\frac{T^{u+v(\varepsilon+i)}}{(1-T)\left(1-T^{\varepsilon+i}\right)}
$$

Then for $0 \leqslant T<1$, we have that

$$
F(T)=\sum_{n_{1}=0}^{\infty} \sum_{n_{2}=0}^{\infty} T^{\left(n_{1}+u\right)+(\varepsilon+i)\left(n_{2}+v\right)}
$$

Thus the zeta function associated with $F(T)$ is given by

$$
Z_{F}(s)=\sum_{n_{1}=0}^{\infty} \sum_{n_{2}=0}^{\infty}\left[\left(n_{1}+u\right)+(\varepsilon+i)\left(n_{2}+v\right)\right]^{-s}, \quad \operatorname{Re} s>2
$$

$Z_{F}(s)$ has its analytic continuation in the whole complex plane and its special values at $s=-2 n$ are given by ([2, Proposition 2])

$$
Z_{F}(-2 n)=\sum_{k=0}^{2 n+2} \frac{(2 n) !}{k !(2 n+2-k) !} B_{k}(u) B_{2 n+2-k}(v)(\varepsilon+i)^{2 n+1-k} .
$$

On the other hand, we also have

$$
Z_{F}(-2 n)=(2 n) ! \frac{1}{2 \pi i} \int_{|z|=\delta} z^{-(2 n+1)} F\left(e^{-z}\right) d z
$$

As $\varepsilon \rightarrow 0$, we get

$$
\begin{aligned}
& \sum_{k=0}^{2 n+2} \frac{B_{k}(u) B_{2 n+2-k}(v)}{k !(2 n+2-k) !}(i)^{2 n+1-k} \\
& =\frac{1}{2 \pi i} \int_{|z|=\delta} \frac{\exp \{-(u+i v) z\} d z}{z^{2 n+1}\left(1-e^{-z}\right)\left(1-e^{-i z}\right)} \\
& =-\sum_{k \neq 0}\left[\text { Residues of } \frac{\exp \{-(u+i v) z\}}{z^{2 n+1}\left(1-e^{-z}\right)\left(1-e^{-i z}\right)} \text { at } z=2 k \pi, 2 k \pi i\right] \\
& =-\sum_{k=1}^{\infty}\left(\frac{1}{2 k \pi}\right)^{2 n+1} \frac{1}{i}\left[\frac{\exp \{-(u+i v) 2 k \pi\}}{1-e^{-2 k \pi}}-\frac{\exp \{(u+i v) 2 k \pi\}}{1-e^{2 k \pi}}\right] \\
& \quad-\sum_{k=1}^{\infty}\left(\frac{1}{2 k \pi i}\right)^{2 n+1}\left[\frac{\exp \{-(u+i v) 2 k \pi i\}}{1-e^{2 k \pi}}-\frac{\exp \{(u+i v) 2 k \pi i\}}{1-e^{-2 k \pi}}\right] .
\end{aligned}
$$


Multiplying by $i$ on both sides gives

$$
\begin{aligned}
& \sum_{k=0}^{2 n+2} \frac{B_{k}(u) B_{2 n+2-k}(v)}{k !(2 n+2-k) !}(i)^{2 n+2-2 k} \\
& =-\sum_{k=1}^{\infty}\left(\frac{1}{2 k \pi}\right)^{2 n+1}\left[\frac{\exp \{-(u+i v) 2 k \pi\}}{\left.1-e^{-2 k \pi}-\frac{\exp (u+i v) 2 k \pi}{1-e^{2 k \pi}}\right]}\right. \\
& \quad+(-1)^{n} \sum_{k=1}^{\infty}\left(\frac{1}{2 k \pi}\right)^{2 n+1}\left[\frac{\exp \{(u+i v) 2 k \pi i\}}{1-e^{-2 k \pi}}-\frac{\exp \{-(u+i v) 2 k \pi i\}}{1-e^{2 k \pi}}\right] .
\end{aligned}
$$

Now set $u=v=0$ and $u=v=1 / 2$. Then our required identities follow since

$$
\operatorname{coth} x=\frac{e^{x}+e^{-x}}{e^{x}-e^{-x}}
$$

and

$$
\operatorname{csch} x=\frac{2}{e^{x}-e^{-x}}
$$

\section{REFERENCES}

[1] B.C. Berndt, Ramanujan's Notebooks Part I and Part II (Springer-Verlag, Berlin, Heidelberg, New York, 1985 and 1989).

[2] M. Eie and K.F. Lai, 'On Bernoulli identities and applications, Part I and II', Rev. Met. Iberoamericana 14 (1998), 167-213.

Department of Mathematics

National Chung Cheng University

Ming-Hsiung

Chia-Yi 621

Taiwan

Republic of China

email: mkeie@math.ccu.edu.tw ylong@alumni.ccu.edu.tw 